\title{
Atrophic gastritis, Epstein-Barr virus infection, and Epstein-Barr virus-associated gastric carcinoma
}

\author{
Yasuharu Kaizaki, Shinji Sakurai, Ja-Mun Chong, and Masashi Fukayama \\ Department of Pathology, Jichi Medical School, 3311-1 Yakushiji, Minamikawachi-machi, Kawachi-gun, Tochigi 329-0498, Japan
}

\begin{abstract}
:
Background. The developmental process of Epstein-Barr virus (EBV)-associated gastric carcinoma (EBVaGC) has not been clearly demonstrated, especially in its relation to intestinal metaplasia and epithelial EBV infection.

Methods. Gastritis and intestinal metaplasia was histologically evaluated in non-neoplastic gastric mucosa that surrounded early carcinoma of EBVaGCs $(n=23)$ and EBV-negative gastric carcinomas (GCs) (intestinal type, $n=139$; diffuse type, $n=44)$. Helicobacter pylori infection was evaluated by immunohistochemistry. EBV infection in the gastric mucosa was examined by both RNA probe in situ hybridization (ISH) and polymerase chain reaction (PCR) for the BamHI$W$ region of $\mathrm{EBV} D N A$, the latter of which was applied to the microdissected mucosa.

Results. Marked grade of atrophy and moderate to marked grade of lymphocytic infiltration were significantly more frequent in EBVaGCs (74\% and $78 \%$, respectively), compared to intestinal-type (49\% and $12 \%)$ and diffuse-type $(27 \%$ and $12 \%$ ) of EBV-negative GCs. Only 13\% of EBVaGCs were surrounded by intestinal metaplasia, in contrast to $41 \%$ of intestinal-type EBV-negative GCs. Immunohistochemistry revealed nearly the same frequencies of $H$. pylori infection $(\mathbf{7 0} \%)$ in three types of GCs. RNA probe ISH for EBV-DNA failed to identify any positive cells in nonneoplastic mucosa, including intestinal metaplasia. Two of 118 microdissected samples of EBVaGC and 5 of 62 samples of EBV-negative GCs showed amplification of EBV-DNA, consisting of 3 pyloric and 4 fundic but no metaplastic gland samples.

Conclusions. EBVaGC may develop from rare EBV-infected epithelial cells with severe atrophic gastritis, but the process is not directly related to intestinal metaplasia or $\boldsymbol{H}$. pylori infection.
\end{abstract}

Key words: Epstein-Barr virus, gastric carcinoma, intestinal metaplasia, atrophic gastritis

Offprint requests to: M. Fukayama

Received for publication on Sept. 22, 1998; accepted on April 27, 1999

\section{Introduction}

Epstein-Barr virus (EBV)-associated gastric carcinoma (EBVaGC) is the most common among various lethal malignancies associated with EBV [1]. EBVaGC consists of two types of carcinomas, most of lymphoepithelioma (LE)-like gastric carcinoma [2] and 10\% or less of ordinary gastric carcinoma. Although the relative frequency of both types depends on the strictness of the histological criteria, LE-like carcinoma appears to constitute one-fifth of EBVaGC. The pathological features of both types have been well described, such as male predominance, location primarily in the proximal stomach, and moderately or poorly differentiated type of histology. However, the developmental process of EBVaGC has yet to be clearly demonstrated. In nonneoplastic gastric mucosa, rarity or complete absence of epithelial cells showing positive signal by EBER (EBVencoded small RNA) with in situ hybridization (ISH) suggests that EBV infection is relatively rare in gastric epithelia [3]. On the other hand, Yanai et al. [4] and Jing et al. [5], using a commercially available DNA ISH kit, recently insisted that EBV infection was occasionally observed in epithelial cells of intestinal metaplasia. However, the signals in the epithelial cells presented in the studies were too strong for the DNA probe ISH without any enhancing procedure, as the copy number of EBV in EBVaGC is generally considered to reach only a few hundred at most [3], the lower limit for detection. Because the methods in both studies lacked sufficient control studies [6], their finding should be interpreted with caution and needs reexamination.

EBVaGCs occur much more frequently as multiple carcinoma than expected if it develops independently $[1,7]$, suggesting that the nonneoplastic mucosa of the proximal stomach bearing EBVaGC has been conditioned to develop EBVaGC (field cancerization). To help reconcile these findings with the recently hypothesized EBV infection in the intestinal metaplasia, we 
attempt here to evaluate the detailed pathology of nonneoplastic mucosa of EBVaGC, and to analyze the EBV infection by ISH of EBV DNA using RNA probes and by polymerase chain reaction (PCR) detection of EBV DNA, which was applied to the microdissected gastric mucosa with or without intestinal metaplasia.

\section{Materials and methods}

Early gastric carcinomas (251) were surgically and/or endoscopically resected from 221 patients at the Jichi Medical School Hospital. The early gastric carcinomas were consecutive cases selected solely on the basis of the depth of invasion. The carcinomatous invasion was within the mucosa in 194 carcinomas (m-carcinoma) and was observed in the submucosa in 57 carcinomas (sm-carcinoma). None of the patients were clinically immunosuppressive. No one had Barrett's esophagus or type A gastritis. There was no specific protocol of Helicobacter pylori eradication therapy for these patients before the resection.

Histological assessment of gastritis and immunohistochemical study of $H$. pylori were comparatively evaluated in three groups of early gastric carcinomas, EBVaGC and intestinal and diffuse types of EBVnegative gastric carcinoma (GC), which were classified according to Lauren [8].

\section{Histological examination}

Pathological examination was performed according to the guidelines of the Japanese Classification of Gastric Carcinoma [9]. The resected stomach was opened along the greater curvature, extended and pinned on a plate, and then fixed in $15 \%$ buffered formalin. The whole lesion of the carcinoma was subjected to routine histological examination by the step-section method. The tissue samples were taken from the early carcinoma as well as the nonneoplastic mucosa, encompassing a length of at least $1 \mathrm{~cm}$ either from the oral or anal edge of the carcinoma.

The localization of the carcinoma relative to the mucosal element was determined by the constituent glands of the surrounding mucosa as the clinically determined localization occasionally failed to reflect the mucosal element because of individual variability and the accompanying inflammatory changes. When both sides of the carcinoma consisted commonly of pyloric gland mucosa and intestinal metaplasia without fundic gland, the localization of the carcinoma was the pyloric gland zone. When the surrounding mucosa consisted of fundic gland mucosa without intestinal metaplasia, the localization was classified as fundic gland zone. When the surrounding mucosa consisted of mixed fundic and py- loric gland mucosa with or without intestinal glands, the localization was classified as intermediate zone. Then, with reference to complete sets of slides covering the whole area of the carcinoma, the following parameters were evaluated in the surrounding nonneoplastic mucosa: (a) atrophy of the mucosa, graded as mild, moderate, and marked; (b) absence or presence of intestinal metaplasia. When intestinal metaplasia was present in either side or both sides of the carcinoma in all slides, it was classified by its distribution relative to the carcinoma, such as anal side, oral side, both sides (i.e., whole circumference), or miscellaneous; (c) lymphocytic infiltration, graded as mild, moderate, or marked. In the present study, we did not evaluate the grade of neutrophilic infiltration because surgery or endoscopic mucosectomy apparently affects the dynamics of neutrophils. The grades of atrophy and lymphocytic infiltration were determined using the visual analog scales of the updated Sydney System [10] to avoid bias of estimation.

\section{In situ hybridization of EBV-encoded RNA}

EBER ISH was applied to the formalin-fixed and paraffin-embedded specimens, as reported previously [3]. Paraffin sections $3 \mu \mathrm{m}$ thick were deparaffinized, rehydrated, and predigested with protease for $15 \mathrm{~min}$ at $37^{\circ} \mathrm{C}$. The sections were then hybridized with a digoxigenin-labeled oligo-probe $(0.5 \mathrm{ng} / \mathrm{ml}$ of hybridization buffer) overnight at $37^{\circ} \mathrm{C}$. After washing in $0.5 \times$ SSC for $30 \mathrm{~min}$, the hybridization signal was detected by serial incubation with an antidigoxigenin antibodyalkaline phosphatase conjugate, and then with 5-bromo4-chloro-3-indolyl phosphate (BCIP) and nitroblue tetrazolium salt (NBT), according to the supplier's protocol (Boerhringer Mannheim, Tokyo, Japan).

\section{Immunohistochemistry of $\mathrm{H}$. pylori}

To assess the frequency of $H$. pylori infection, we applied immunohistochemistry to the formalin-fixed paraffin-embedded sections of nonneoplastic mucosa. Because of the retrospective nature of the present study, the cases examined were selected so that the sufficient length of the mucosae from both fundic and pyloric zone could be evaluated: 14 cases from EBVaGC, and 14 and 10 cases from intestinal and diffuse types of EBV-negative GC. As for the immunohistochemistry, the sections were pretreated by trypsin at $37^{\circ} \mathrm{C}$ for $30 \mathrm{~min}$. The sections were incubated with a rabbit anti-H. pylori antibody (Dakopatts, Glostrop, Denmark) at a dilution of $1: 50$ at $4{ }^{\circ} \mathrm{C}$ overnight. Then, the avidin-biotin-horseradish peroxidase method was applied using a Vectastain Elite ABC Kit (Vector, Burlingame, CA, USA), and the peroxidase 
reaction was developed by incubation with 3-, 3'diaminobenzidine.

\section{In situ hybridization of EBV DNA}

ISH of EBV DNA was performed to evaluate the possibility that there may be EBV-infected epithelial cells lacking expression of EBER. RNA probes instead of DNA probes were used in the present study because they are generally much more sensitive. Furthermore, the specificity of hybridization can be enhanced by RNase treatment, which digests unreacted RNA probes. Sections $3 \mu \mathrm{m}$ thick were cut from formalinfixed and paraffin-embedded stomach tissue showing no histological evidence of neoplastic lesions from five cases with GC. The sections of EBVaGC were used as a positive control. After dewaxing with xylene, incubation in ethanol, and air-drying, the sections were digested for $15 \mathrm{~min}$ with proteinase K (Sigma, Tokyo, Japan) at a concentration of $1 \mathrm{mg} / \mathrm{ml}$ in distilled water. Next, after thorough rinsing in distilled water, dehydration through a graded alcohol series, and air-drying, the sections were denatured on a hot plate at $97^{\circ} \mathrm{C}$ for five minutes and hybridized at $37^{\circ} \mathrm{C}$ overnight.

The RNA probe for EBV DNA was kindly provided by Dr. K. Takada (Hokkaido University School of Medicine). The PstI subfragment of the BamHI-W fragment of EBV DNA (at nucleotides 16287 to 16535) had been subcloned into the PstI site of the pBSII $\mathrm{KS}+$ vector (Stratagene, La Jolla, CA, USA), and the digoxigenin-labeled RNA probe was generated by in vitro transcription using T7 RNA polymerase (DIG RNA labeling kit SP6/7; Boehringer Mannheim). The hybridization solution consisted of $50 \%$ formamide, $10 \%$ dextran sulfate, $3 \times$ SSC $(0.45 \mathrm{M}$ sodium chloride, $0.045 \mathrm{M}$ sodium citrate), $20 \mathrm{mM}$ sodium phosphate, $\mathrm{pH} 7.4,1 \times$ Denhardt solution, $100 \mathrm{mg} / \mathrm{ml}$ salmon sperm DNA, $125 \mu \mathrm{g} / \mathrm{ml}$ yeast tRNA, and $3 \mu \mathrm{g} / \mathrm{ml}$ digoxigeninlabeled probe. After hybridization, the coverslips were removed in washing buffer $(50 \mathrm{mM}$ Tris- $\mathrm{HCl}, 150 \mathrm{mM}$ sodium chloride, $0.1 \%$ Triton $\mathrm{X}-100, \mathrm{pH} 7.6$ ).

To exclude nonspecific binding of the RNA probes, the sections were digested with RNase after ISH with the RNA probe. The sections with RNase treatment were digested for $30 \mathrm{~min}$ at $37^{\circ} \mathrm{C}$ with RNase A (Sigma) at a concentration of $50 \mathrm{mg} / \mathrm{ml}$ in NTE $(500 \mathrm{mM}$ sodium chloride, $10 \mathrm{mM}$ Tris- $\mathrm{HCl}, 1 \mathrm{mM}$ EDTA, pH 7.50). Then, the sections with or without RNase treatment were washed twice for $30 \mathrm{~min}$ each in $0.1 \times$ SSC containing $2 \mathrm{mM}$ magnesium chloride, $0.05 \%$ bovine serum alloumin (BSA), and $0.1 \%$ Triton $\mathrm{X}-100$ at $57^{\circ} \mathrm{C}$. Hybridization signal was detected by antidigoxigenin antibody-peroxidase conjugate (Boehringer Mannheim). To amplify the hybridization signal, we used the catalyzed reporter deposition (CARD) procedure accord- ing to Kerstens et al. [11] and the benzidine reaction for coloration.

\section{PCR detection of EBV DNA in microdissected tissue samples}

To confirm the observation of ISH, we also evaluated the presence of EBV in epithelial glands, which were microdissected directly from the paraffin-embedded sections of nonneoplastic mucosa. The nonneoplastic mucosa of 15 early EBVaGCs and 4 EBV-negative GCs were examined. Referring to the microscopical observation of the hematoxylin-stained section, a limited area of nonneoplastic mucosa was microdissected from the section with a needle under a microscope. The microdissected areas were confined in the mucosa, two to three glands in width. Then, the microdissected tissue was digested in $20 \mu \mathrm{l}$ of extraction buffer $[50 \mathrm{mmol} / \mathrm{L}$ Tris- $\mathrm{HCl}$ buffer ( $\mathrm{pH} 8.5$ ) containing $1 \mathrm{mmol} / \mathrm{L}$ EDTA, $10 \mathrm{mg} / \mathrm{mL}$ proteinase $\mathrm{K}$, and $0.5 \%$ Tween 20] [12]. After incubation for $8 \mathrm{~h}$ at $62{ }^{\circ} \mathrm{C}$, samples were boiled for $10 \mathrm{~min}$ to inactivate proteinase $\mathrm{K} ; 1 \mu \mathrm{l}$ of the liquid phase was used for PCR.

The Bam HI-W region was amplified by a nested PCR technique [13]. For the first PCR, the primer set $(0.6 \mathrm{mM})$ of B-1 (5'-AAAGCGGGTGCAGTAACAGGTAAT-3', B95-8 13971-13994) and B-2 (5'-TTGACTGAGAAGGTGGCCTAGCAA-3', 14286-14263) was used with $2.5 \mathrm{U} / 100 \mathrm{ml}$ Taq DNA polymerase (Takara, Otsu, Japan) in a reaction buffer $(0.2 \mathrm{mM}$ $\mathrm{dNTP}, 50 \mathrm{mM} \mathrm{KCl}, 10 \mathrm{mM}$ Tris- $\mathrm{HCl}$, and $1.5 \mathrm{mM}$ $\mathrm{MgCl}_{2}$ ). PCR was carried out by 30 cycles, each consisting of $30 \mathrm{~s}$ of denaturing at $95^{\circ} \mathrm{C}, 30 \mathrm{~s}$ of annealing at $54^{\circ} \mathrm{C}$, and $2 \mathrm{~min}$ of extension at $76^{\circ} \mathrm{C}$. Then, $1 \mu \mathrm{l}$ of the first PCR product was used as a template for the second PCR, which was performed under the same conditions as the first PCR, except for using the primer sets, B-3 (5'-GATTTGGACCCGAAATCTGA-3', 1400514014) and B-4 (5'-TCTGGGGGCTTATTCCTCTT$\left.3^{\prime}, 14205-14186\right)$; the size of the amplified fragment was $201 \mathrm{bp}$. For the control to check the validity of the template DNA, the Ki-ras gene was similarly amplified by the nested PCR method that was reported previously (the size of the amplified fragment was $117 \mathrm{bp}$ ) [14]. Second PCR products of the Bam HI-W region and Kiras genes were electrophoresed and then stained with ethidium bromide and photographed under ultraviolet light.

\section{Statistical analysis}

Statistical analyses were performed using Student's $t$ test, Fisher's exact test, and $\chi^{2}$ analysis with Yates' correction. A probability of less than $5 \%$ was interpreted as significant. 
Table 1. Clinicopathological features and mucosal characteristics of EBV-associated (EBVaGC) and EBV-negative gastric carcinomas

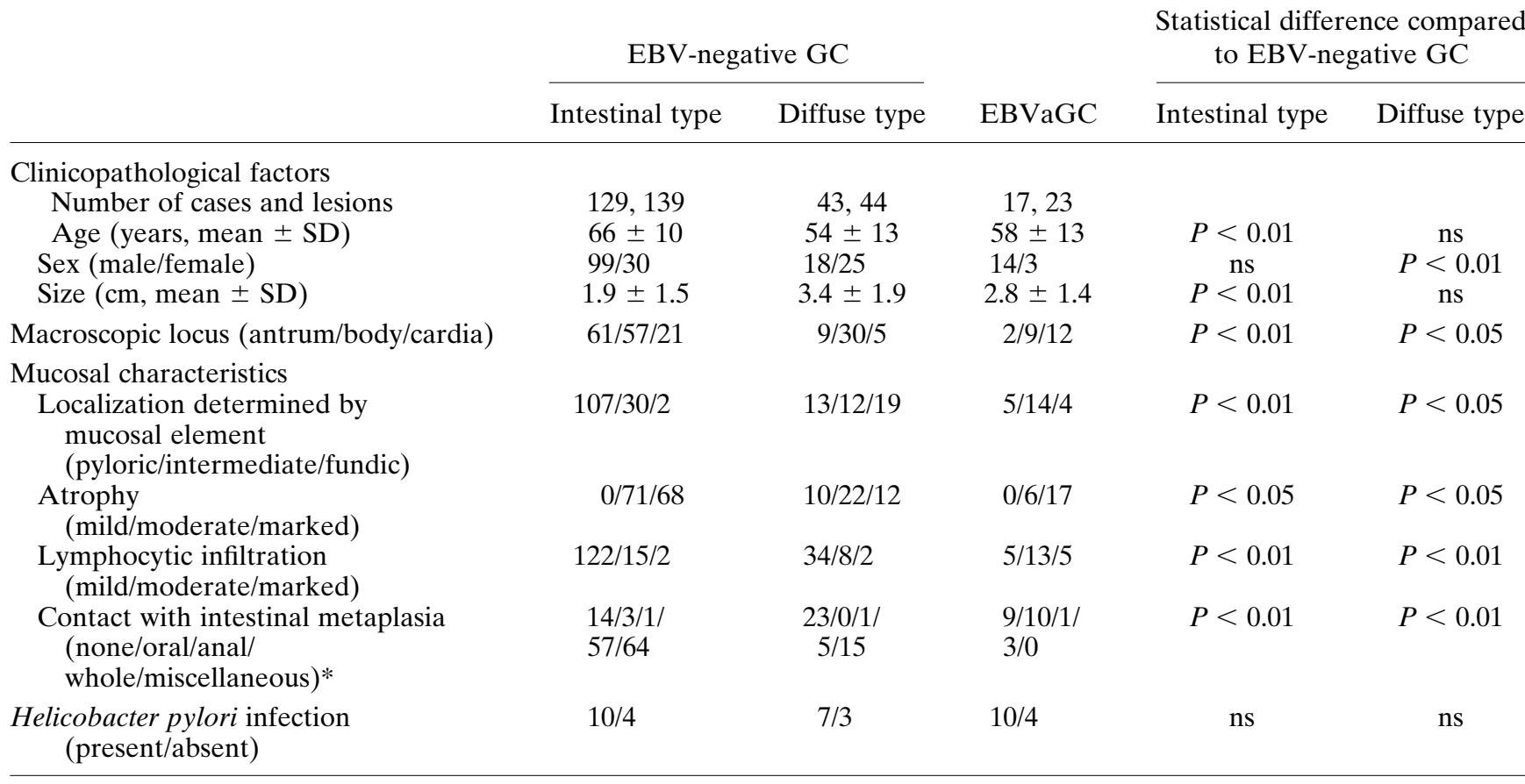

EBV, Epstein-Barr virus; GC, gastric carcinoma. *, Presence of intestinal metaplasia; oral or anal means that intestinal metaplasia is present only in the oral or anal side of cancer lesion

\section{Results}

\section{Clinical and histological features of EBVaGC}

With a highly sensitive EBER ISH, 23 carcinomas showed positive signals (EBVaGC) in 251 early gastric carcinomas $(9.2 \%)$. Nearly all the carcinoma cells showed positive signal in the nuclei whenever any positivity was observed in the carcinoma. These EBVaGC $(11 \mathrm{~m}$ - and $12 \mathrm{sm}$-carcinomas) were the subjects in the present study, as well as $183 \mathrm{~m}$-carcinomas of EBVnegative GC (139 intestinal- and 44 diffuse-type). As for the histology of EBVaGC, 2 of $12 \mathrm{sm}$-carcinomas of EBVaGC were LE-like carcinomas and 5 of them contained partially LE-like histology.

EBVaGC in its early stage had several clinical features compared to the intestinal or diffuse type of EBVnegative GC (Table 1). The patients with EBVaGC were younger (mean, $58 \pm 13$ years old) than the ones with intestinal-type EBV-negative GC (mean, $66 \pm 10$ ). A male predominance in EBVaGC (male:female ratio, $14: 3)$ was contrasted to an equal occurrence of diffusetype of EBV-negative GC $(18: 25)$. The size of the carcinoma was larger in EBVaGC than in the intestinal type but was comparable with that in diffuse-type EBVnegative GC. When the locus of the carcinoma was determined clinically or macroscopically, EBVaGCs occurred preferentially in the proximal stomach, espe- cially in the cardia $(12 / 23,52 \%)$, compared to intestinaltype $(21 / 139,15 \%)$ and diffuse-type EBV-negative GCs $(5 / 44,11 \%)$.

\section{Histological evaluation of atrophic gastritis}

The distribution of the mucosal element is affected by individual variability and gastritis. When the localization of the carcinoma was determined with reference to the constituent glands in the surrounding mucosa (Table 1), 14 of 23 early EBVaGC (61\%) were situated at the intermediate zone. On the other hand, the intestinal-type EBV-negative GC were predominantly within pyloric gland mucosa $(107 / 139,77 \%)$, and the diffuse type occurred equally in three zones.

Among the parameters of pathological changes in the surrounding mucosa, a marked grade of atrophy was observed in $74 \%$ of EBVaGC (17/23) (Fig. 1), in contrast to only $49 \%$ of intestinal-type $(68 / 139)(P<0.05)$ and $27 \%$ of diffuse-type (12/44) EBV-negative GC $(P<$ $0.01)$. Similarly, lymphocytic infiltration with a moderate or marked grade was observed in 18 of 23 EBVaGC $(78 \%)$, in contrast to only 17 of 139 intestinal-type $(12 \%)(P<0.01)$ and 10 of 44 diffuse-type $(23 \%)$ EBVnegative GC $(P<0.01)$. In 9 of 23 lesions $(39 \%)$, there was no intestinal metaplasia adjacent to the EBVaGC, and in 10 of 23 lesions (43\%), intestinal metaplasia was 


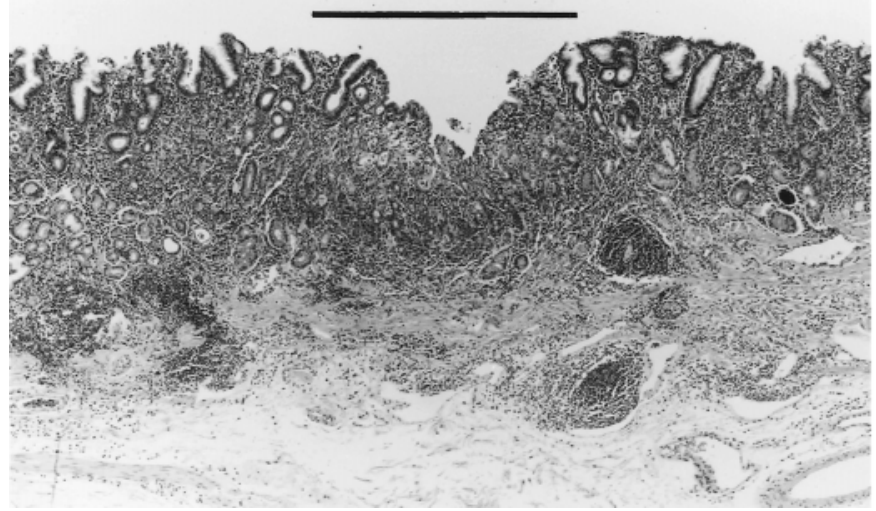

Fig. 1a,b. Histological features of intramucosal Epstein-Barr virus-associated gastric carcinoma (EBVaGC) and its surrounding nonneoplastic mucosa. a Low-power view of intramucosal stage of $\mathrm{EBVaGC}$, which is present in a limited area of the mucosa (bar). Note paucity of glands in the nonneoplastic mucosa. There is marked lymphocytic infiltration, but no intestinal metaplasia around the carcinoma

found only in the anal side of the mucosa. Only 3 carcinomas $(13 \%)$ were fully surrounded by intestinal metaplasia. On the other hand, intestinal-type EBV-negative GC were frequently accompanied by intestinal metaplasia in the whole circumferance of the carcinoma (57/139, $41 \%)(P<0.01)$.

\section{Immunohistochemical evaluation of $\mathrm{H}$. pylori}

Immunohistochemistry with benzidine reaction clearly demonstrated $H$. pylori as brown rods or commas in the mucus layer or at the luminal surface of the epithelial cells of the gastric pit, even if the number of positive organisms was very small. The frequencies of $H$. pylori infection are nearly the same in the nonneoplastic mucosa of the three types of GCs (Table 1): 71\% (10/14 cases) in $\mathrm{EBVaGC}$, and $71 \%(10 / 14)$ and $70 \%(7 / 10)$ in intestinal- and diffuse-type EBV-negative GC, respectively.

\section{ISH evaluation of EBV infection in nonneoplastic mucosa}

In our present findings on nonneoplastic mucosa of the stomach in a limited length, there were no epithelial cells showing positive signal by EBER ISH in the vicinity of the carcinoma.

By use of antisense RNA probe targeting the BamHIW region of EBV DNA, most of the cancer cells of EBVaGC showed positive signals depicted as positive dots studding the nuclei. These positive signals remained within the nuclei of carcinoma cells with RNase

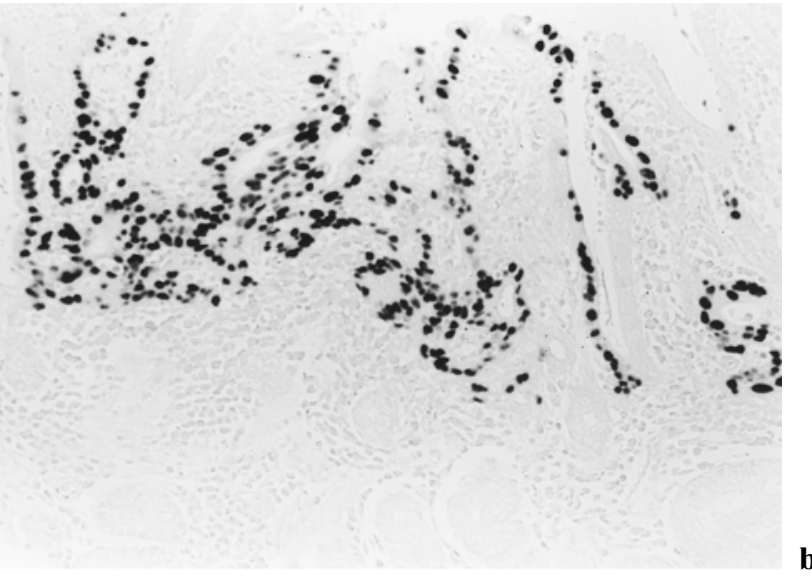

b Nearly all the carcinoma cells show a positive signal for EBER (EBV-encoded small RNA) in the nuclei of intramucosal EBVaGC. The carcinoma, classified as moderately differentiated tubular adenocarcinoma, consists of branching of abortive glands at the middle of the proper mucosa. Nonneoplastic pyloric glands, negative for EBER ISH, remain below the carcinoma

treatment (Fig. 2a). In the nonneoplastic gastric mucosa $(n=5)$ without RNase treatment, strong positive signals were observed in some nuclei of epithelial cells of the gastric mucosa including intestinal metaplasia or lymphocytes infiltrating to the lamina propria mucosae. However, with RNase treatment, positive signals observed in nonneoplastic mucosa were completely abolished (Fig. 2b).

\section{PCR evaluation of nonneoplastic mucosa}

To confirm the observation by ISH, we also evaluated the presence of EBV in epithelial glands microdissected directly from the paraffin-embedded sections of nonneoplastic mucosa (Fig. 3) of different portions of the stomach. Figure 3 a shows a representative case in which 34 minute foci were micodissected from the formalinfixed and paraffin-embedded sections.

Using a nested PCR method, a 117-bp fragment of the Ki-ras gene was successfully amplified in all 180 specimens from the nonneoplastic mucosa (118 samples from 15 cases of EBVaGC and 62 samples from 4 cases of EBV-negative GC). The BamHI-W region of EBV DNA was amplified only in 7 of 180 samples (2 of 118 and 5 of 16 samples of nonneoplastic mucosa of EBVaGC and EBV-negative GC, respectively) (Table 2). These positive samples were derived from 2 cases of EBVaGC and 1 case of EBV-negative GC.

When correlating with the type of gland, 3 of 7 samples with EBV DNA were pyloric or pseudopyloric and the other 4 samples were fundic glands. EBV DNA was not amplified in any of the samples microdissected 

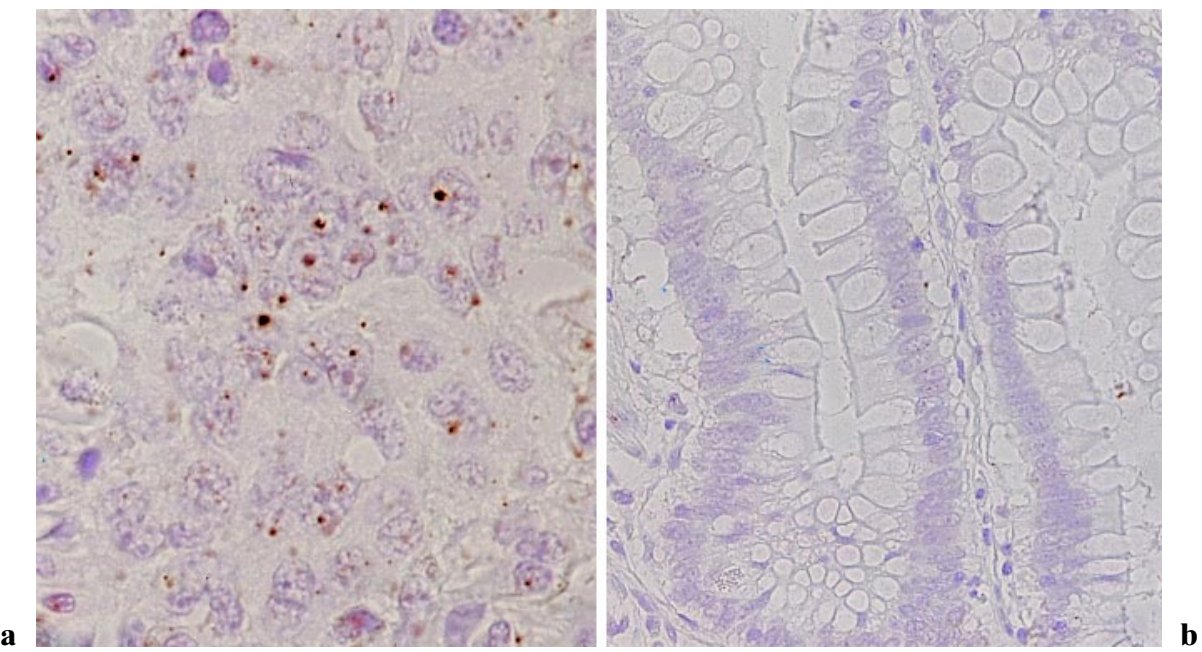

Fig. 2a,b. In situ hybridization evaluation of EBV DNA in EBVaGC and nonneoplastic mucosa. a Using an RNA probe for PstI subfragment of BamHI-W fragment of EBV DNA, most of the carcinoma cells show positive signals with RNase treatment, which appear as positive dots within the nuclei. RNase treatment digests unhybridized RNA probe in the tissue and is efficient to avoid the nonspecific binding of the probe that can occur with DNA probes. b In the nonneoplastic gastric mucosa, there were no epithelial cells positive for EBV DNA with RNase treatment

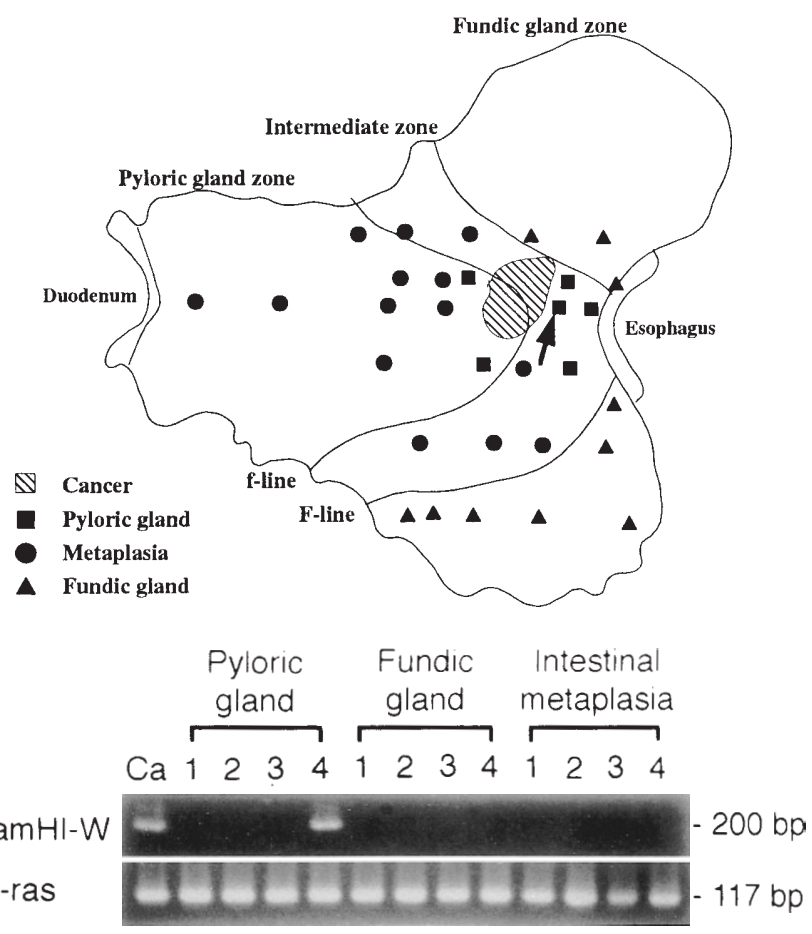

Fig. 3. PCR evaluation of EBV DNA in nonneoplastic mucosa. To assess the distribution of EBV infection in the nonneoplastic mucosa, the presence of the Bam HI-W region was evaluated in different minute foci in the resected stomach. Top: Map shows the microdissected foci in the resected stomach with an intramucosal EBVaGC located at the intermediate zone. The $f$ line and $F$ line are borders between the intermediate zone and pyloric and fundic zones, respectively. The BamHI-W region of EBV DNA was amplified by PCR in only 1 of 34 samples microdissected from the nonneoplastic mucosa (arrow). Bottom: Using a nested PCR method, only 1 of the pyloric gland samples microdissected from the nonneoplastic mucosa shows amplification of the BamHI-W region; all samples show amplification of the Ki-ras gene from intestinal metaplasia. EBV infection was detected in 4 of 59 samples from the intermediate zone and in 3 of 57 from the fundic gland mucosa. In contrast, it was not observed in any samples from the pyloric gland mucosa, suggesting that the pyloric gland samples with EBV DNA consisted of pseudopyloric glands rather than genuine pyloric glands.

\section{Discussion}

EBVaGC is a unique type of gastric carcinoma in which EBV is closely associated with its development [1]. Clinical features of $\mathrm{EBVaGC}$ have been pointed out, such as male predominance and the preference in the proximal stomach. In the present study, which focused on inflammatory and metaplastic changes in the nonneoplastic mucosa, stomachs with EBVaGC also demonstrated several features: EBVaGC was predominantly localized within the intermediate zone, and both gastric atrophy and chronic inflammation were significantly more severe in EBVaGC than that with the intestinal or diffuse type of EBV-negative GC. As the frequences of $H$. pylori infection were nearly the same in the nonneoplastic mucosa of three types of gastric carcinomas, a specific type of severe gastritis itself, rather than the H. pylori infection, may be a predisposing factor for the development of EBVaGC.

As for the relation between the intestinal metaplasia and EBVaGC, there has been some controversy. A few researchers recently described that EBV infection occasionally occurred in the epithelial cells of the intestinal metaplasia $[4,5]$. We do not think that this is true. First, in the present study, the relation of intestinal metaplasia with EBVaGC was not so close as that with the intestinal-type EBV-negative GC. Second, we could not 
Table 2. PCR detection of EBV-BamHI-W in microdissected nonneoplastic mucosa of early EBV associated and EBV-negative gastric carcinomas

\begin{tabular}{|c|c|c|c|c|}
\hline & \multicolumn{2}{|c|}{ EBV-negative GC } & \multirow[b]{2}{*}{ EBVaGC } & \multirow[b]{2}{*}{ Total } \\
\hline & Intestinal type & Diffuse type & & \\
\hline \multicolumn{5}{|l|}{ Site of sampling } \\
\hline Pyloric gland mucosa & $0 / 13(0 / 2)$ & $0 / 15(0 / 2)$ & $0 / 36(0 / 8)$ & $0 / 62(0 / 12)$ \\
\hline Intermediate zone & $3 / 14(1 / 2)$ & $0 / 7(0 / 2)$ & $1 / 38(1 / 11)$ & $4 / 59(2 / 15)$ \\
\hline Fundic gland mucosa & $2 / 5(1 / 2)$ & $0 / 8(0 / 2)$ & $1 / 44(1 / 11)$ & $3 / 57(2 / 15)$ \\
\hline \multicolumn{5}{|l|}{ Gland type of the sample } \\
\hline Pyloric or pseudopyloric & $2 / 11(1 / 2)$ & $0 / 13(0 / 2)$ & $1 / 29(1 / 8)$ & $3 / 53(2 / 12)$ \\
\hline Fundic & $3 / 7(1 / 2)$ & $0 / 15(0 / 2)$ & $1 / 52(1 / 11)$ & $4 / 74(2 / 15)$ \\
\hline Intestinal metaplasia & $0 / 14(0 / 2)$ & $0 / 2(0 / 2)$ & 0/37 (0/10) & $0 / 53(0 / 14)$ \\
\hline Total & $5 / 32(1 / 2)$ & $0 / 30(0 / 2)$ & $2 / 118(2 / 15)$ & $7 / 180(3 / 19)$ \\
\hline
\end{tabular}

confirm their ISH finding using the probe of the same source and the same protocol (data not shown) or using a more sensitive but strict method of ISH with an RNA probe. Third, EBV-DNA was not amplified from the microdissected samples of the metaplastic glands. Although the microdissected tissues contained not only epithelial cells but possibly some interstitial cells and lymphocytes, we can assume that the detection rate roughly reflected the infection status of EBV in the gastric mucosa. Thus, these results in the present study clearly demonstrated that EBV infection is a rare event in the gastric mucosa, and that the primary target of EBV infection is not the epithelial cells of the metaplastic glands. The conclusion is compatible with the following facts in EBVaGC. The mucus of EBVaGC shares characteristics with that of the gastric gland [15], and the genetic abnormalities found in intestinal-type EBV negative GC are extremely rare in EBVaGC [16].

The nonneoplastic mucosa of the proximal stomach bearing EBVaGC has been conditioned to develop EBVaGC [6], and the present study demonstrated that one of characteristics of the mucosa was severe atrophic gastritis. Then, the question is how atrophic gastritis can facilitate the development of EBVaGC. Imai et al. [17] demonstrated that cell-to-cell contact dramatically facilitates the EBV infection of the epithelial cell lines in an in vitro experimental system using a genetically engineered EBV [18]. Along the line, dense infiltration of lymphocytes in the nonneoplastic mucosa of EBVaGC may increase the frequency of cell-to-cell contact of EBV-producing lymphocytes with fundic glands or pseudopyloric glands of intermediate zone. Alternatively, atrophic gastritis may specifically augment the genetic changes in the EBV-infected epithelial cells or interrupt the immunological exclusion of the immortalized epithelial cells. A similar close relationship between chronic inflammation and EBV-associated neoplasm has been demonstrated in pyothorax- associated lymphoma $[19,20]$. An experimental system of in vitro EBV infection of the gastric epithelial cells, not the epithelium of intestinal metaplasia, will clarify the process more precisely.

Acknowledgments. This work was supported by a grantin aid for scientific research on priority areas (09253103) from the Ministry of Education, Science, Sports, and Culture of Japan.

\section{References}

1. Fukayama M, Chong J-M, Kaizaki Y. Epstein-Barr virus and gastric carcinoma. Gastric Cancer 1998;1:104-14.

2. Nakamura S, Ueki T, Yao T, Ueyama T, Tsuneyoshi M. EpsteinBarr virus in gastric carcinoma with lymphoid stroma. Special reference to its detection by the polymerase chain reaction and in situ hybridization in 99 tumors, including a morphologic analysis. Cancer (Phila) 1994;73:2239-49.

3. Fukayama M, Hayashi Y, Iwasaki Y, Chong J-M, Ooba T, Takizawa T, et al. Epstein-Barr virus-associated gastric carcinoma and Epstein-Barr virus infection of the stomach. Lab Invest 1994;71:73-81.

4. Yanai H, Takada K, Shimizu N, Mizugaki Y, Tada M, Okita K. Epstein-Barr virus infection in non-carcinomatous gastric epithelium. J Pathol 1997;183:293-8.

5. Jing X, Nakamura Y, Nakamura M, Yokoi T, Shan L, Taniguchi E, et al. Detection of Epstein-Barr virus DNA in gastric carcinoma with lymphoid stroma. Viral Immunol 1997;10:49-58.

6. Pagani A, Cerrato M, Bussolati G. Nonspecific in situ hybridization reaction in neuroendocrine cells and tumors of the gastrointestinal tract using oligonucleotide probes. Diagn Mol Pathol 1993;2:125-30.

7. Matsunou H, Konishi F, Hori H, Ikeda T, Sasaki K, Hirose Y, et al. Characteristics of Epstein-Barr virus-associated gastric carcinoma with lymphoid stroma in Japan. Cancer (Phila) 1996;77: 1998-2004.

8. Lauren P. The two histological main types of gastric carcinoma: diffuse and so-called intestinal type carcinoma. Acta Pathol Microbiol Scand (A) 1965;64:31-49.

9. The Japanese Research Society for Gastric Cancer. Japanese classification of gastric carcinoma. First English edition. Tokyo: Kanehara, 1995. 
10. Dixon MF, Genta RM, Yardley JH, Correa P. and participants in the International Workshop on the Histopathology of Gastritis, Houston 1994. Classification and grading of gastritis. The updated Sydney System. Am J Surg Pathol 1996;20:116181.

11. Kerstens HMJ, Poddighe PJ, Hanselaar AGJM. A novel in situ hybridization signal amplification method based on the deposition of biotinylated tyramine. J Histochem Cytochem 1995;43:34752.

12. Sakurai S, Sano T, Nakajima T. Clinicopathological and molecular biological studies of gastric adenomas with special reference to p53 abnormality. Pathol Int 1995;45:51-7.

13. Pan L, Diss TC, Peng H, Isaacson PG. Immersion-histo polymerase chain reaction. A practical tool for visualization of single-copy genes in tissue sections. Am J Pathol 1997;150:124552.

14. Fukushima N, Suzuki M, Fukayama M, Analysis of K-ras oncogene mutation directly applied to atypical cell clusters on cytologic smear slides of bile and pancreatic juice. Pathol Int 1998; 48:33-40.
15. Tashiro Y, Arikawa J, Itoh T, Tokunaga M. Clinico-pathological findings of Epstein-Barr virus-related gastric cancer. Gann Monogr Cancer Res 1998;45:87-97.

16. Chong J-M, Fukayama M, Hayashi Y, Takizawa T, Moike M, Konishi M, et al. Microsatellite instability in the progression of gastric carcinoma. Cancer Res 1994;54:4595-7.

17. Imai S, Nishikawa J, Takada K. Cell-to-cell contact as an efficient mode of Epstein-Barr virus infection of diverse human epithelial cells. J Virol 1998;72:4371-8.

18. Yoshiyama H, Imai S, Shimizu N, Takada K. Epstein-Barr virus infection of human gastric carcinoma cells: implication of the existence of a new virus receptor different from CD21. J Virol 1997;71:5688-91.

19. Fukayama M, Ibuka T, Hayashi Y, Ooba T, Koike M, Mizutani S. Epstein-Barr virus in pyothorax-associated pleural lymphoma. Am J Pathol 1993;143:1044-9.

20. Fukayama M, Hayashi Y, Ooba T, Funata N, Ibuka T, Koike M, et al. Pyothorax-associated lymphoma: development of EpsteinBarr virus-associated lymphoma within the inflammatory cavity. Pathol Int 1995;45:825-31. 